\title{
User Adaptable Robot Behavior
}

\author{
Adriana Tapus and Amir Aly \\ Cognitive Robotics Lab \\ ENSTA-ParisTech \\ Paris, 75015, France \\ \{adriana.tapus; amir.aly\}@ensta-paristech.fr
}

\section{SHORT PAPER}

\begin{abstract}
A social intelligent robot should be capable of observing and understanding the changes in the environment so as to behave in a proper manner. It also needs to take into account user preferences, user disability level, and user profile. This paper presents a research work based on socially assistive robotics (SAR) technology that aims at providing affordable personalized physical and cognitive assistance, motivation, and companionship to users. The work described here tries to validate that a robotic system can adapt its behavior to the user profile.
\end{abstract}

KEYWORDS: socially assistive robotics, user customized robot behavior, learning and adaptation

\section{INTRODUCTION}

Research into Human-Robot Interaction (HRI) for socially assistive applications is in its infancy. Recently, there has been a growth of interest in assistive technologies featuring flexible and customizable robotic systems. The main goal of such robots is to support elderly individuals and/or individuals with disabilities in their home environments, and therefore to improve their quality of life.

Cognitive and physical impairments are the major health problems facing elderly people in the new millennium. Age-related cognitive and physical decline affects numerous aspects of a person's health and quality of life [2]. Decline in cognitive and physical abilities has been shown to lead to an increased risk of difficulty in

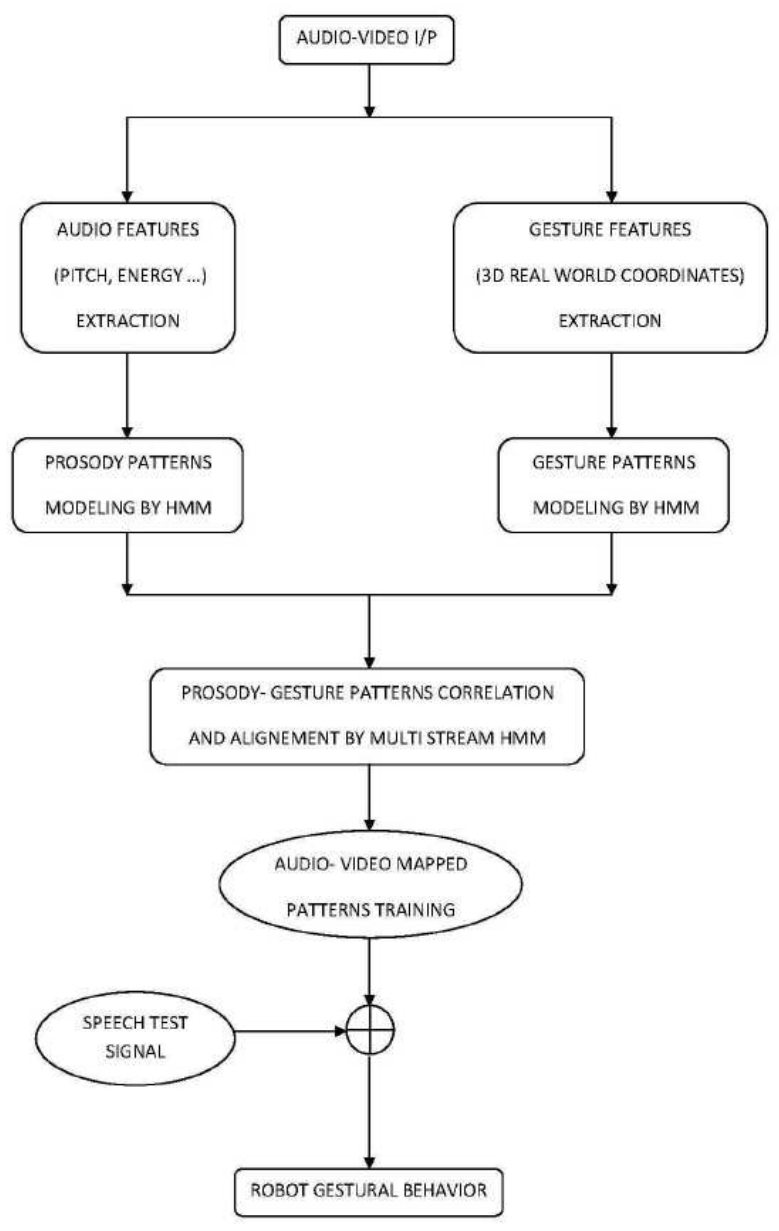

Figure 1. Diagram of the Multi Stream HMM 
performing instrumental activities of daily living (IADL).

Some of these people will may not be able to communicate verbally or perform normal activities, such as getting dressed, eating, and toiletry. Therefore, most affected individuals need some kind of assistance.

In this paper, we present a methodology based on socially assistive robotics (SAR) [3], [7], [8], [9] technology that aims at providing affordable personalized physical and cognitive assistance, motivation, and companionship to users suffering from physical and/or cognitive changes related to aging. The robot is used as customized complement to humans/nurses/therapists. The work described here aims to validate that a robotic system can adapt its behavior to the user profile.

\section{CUSTOMIZED ROBOT BEHAVIOR}

Creating robotic systems capable of adapting their behavior to user personality, user preferences, user profile, and user disability level (physical/cognitive) in order to provide an engaging and motivating customized protocol, is a challenging task. In our previous work [8], [9] we have developed various robotic systems for people who have suffered a stroke and people with Alzheimer's disease and showed the importance of personalized robotic interaction. The robot was able to learn (using online PGRL) and change its behavior to fit the user's personality, preferences, and the level of physical and/or cognitive impairment. The purpose of this adjustment was to be in better agreement with the users and to best help them to maximize their performance in physical tasks and/or cognitive stimulation.

Different perceptual modalities are used by the robot so as to adapt its behavior. We strongly believe that a socially assistive robot that works and interacts with humans and operates among them should have a clear understanding of humans acting in its visual range. Therefore, the robot must have capabilities to recognize and perceive the human and reason on his/her activity and internal state. It was shown in [5] that speech and gesture have an intricate rhythmic relationship. However, gesture and voice based applications constitute a big challenge in human-robot interaction. Most of the related works that try to map the speech with gestures are done in the fields of technologies for interaction in virtual worlds and character animation, e.g., [10], [4].

\section{STEREO VIDEO} 3D CONSTRUCTION EULER ANGLES CALCULATION

Figure 2. Head Gestures and Angles Analysis
In order to have a more natural and adaptable behavior, we developed a learning algorithm that allow the robot to gesticulate properly based on the prosody of the communicating human voice. Both gesture and voice features are modeled using separate Hidden Markov Models (HMM). Voice features are based on the statistical measuring (e.g., mean, variance, max, min, range) of the pitch and energy of voice signal, which could indicate the emotional internal state of the interacting human. The same previous acoustic features beside others (e.g., formants and Mel-frequency cepstral coefficients) are useful in indicting also the gender of the interacting human [1]. The gesture-prosody modeled patterns (see Figure 2) are inter-correlated in order to detect the audio-visual mapping models via multi-stream parallel HMM structure (see Figure 1). The detected audio-visual models are used to estimate a specific gestural behavior according to the incoming voice signal's prosody of the human interacting with the robot.

\section{TRAINING DATABASE}

For the training and testing we used the MVGL-MASAL gesture-speech Turkish database [6]. It contains the audiovisual information of different subjects instructed to tell stories to children audience. We use one part of the database for the training of the models and the other part for the testing.

\section{RESULTS}

This is still ongoing research. The first preliminary results are promising. More will be reported by the time of the workshop.

\section{ACKNOWLEDGEMENTS}

This work is supported by the French National Research Agency (ANR) through Chaire d'Excellence program 2009 (Human-Robot Interaction for Assistive Applications).

\section{REFERENCES}

[1] A. Aly and A. Tapus, "Towards an online voice-based gender and internal state detection model", In Proceedings of the ACM/IEEE Human-Robot Interaction Conference (HRI) - Late Break Paper, Lausanne, Switzerland, March 2011.

[2] Hayden B. Bosworth and K. Warner Schaie, "The relationship of social environment, social networks, and health outcomes in the seattle longitudinal study: Two analytical approaches", Journal of Gerontology, 52B(5):197-205, 1997. 
[3] D. Feil-Seifer and M. J. Matarić, "Defining socially assistive robotics", In Proc. IEEE International Conference on Rehabilitation Robotics (ICORR'05), pages 465-468, Chicago, Il, USA, June 2005.

[4] S. Levine, C. Theobalt, and V. Koltun, "Real-time prosody-driven synthesis of body language", In SIGGRAPH Asia'09: ACM SIGGRAPH Asia 2009, New York, USA, 2009.

[5] D. Loehr, "Aspects of rhythm in gesture and speech", Gesture, 7(2):179-214, 2007.

[6] M.E. Sargn, Y. Yemez, E. Erzin, and A.M. Tekalp, "Analysis of head gesture and prosody patterns for prosody driven head-gesture animation", IEEE Transactions on Pattern Analysis and Machine Intelligence, 30(8):1330-1345, 2008.

[7] A. Tapus and M. J. Matarić, "Emulating empathy in socially assistive robotics," In Proceedings of the American Association of Artificial Intelligence (AAAI) Spring Symposium on - Multidisciplinary Collaboration for Socially Assistive Robotics, Palo Alto,USA, March 2004.
[8] A. Tapus and M. J. Matarić, "Socially assistive robotic music therapist for maintaining attention of older adults with cognitive impairments", In Proceedings of AAAI Fall Symposium AI in Eldercare: New Solutions to Old Problem, Washington D.C., USA, November 2008.

[9] A. Tapus, C. Tapus, and Maja J Matarić, "User-robot personality matching and robot behavior adaptation for post-stroke rehabilitation therapy", Intelligent Service Robotics, 1(2):169-183, April 2008.

[10]L. Valbonesi, R. Ansari, D. McNeill, F. Quek, K. E. M. Duncan, and R. Bryll, "Multimodal signal analysis of prosody and hand motion: Temporal correlation of speech and gestures", In EUSIPCO'02, volume 1, pages 75-78, 2002. 